\title{
André Leroi-Gourhan et Pierre Francastel
}

Alain Schnapp et Pierre Lemonnier

\section{(2) OpenEdition}

\section{Journals}

Édition électronique

URL : http://journals.openedition.org/actesbranly/80

DOI : $10.4000 /$ actesbranly.80

ISSN : 2105-2735

Éditeur

Musée du quai Branly Jacques Chirac

Référence électronique

Alain Schnapp et Pierre Lemonnier, « André Leroi-Gourhan et Pierre Francastel », Les actes de colloques du musée du quai Branly Jacques Chirac [En ligne], 1 | 2009, mis en ligne le 28 juillet 2009, consulté le 08 septembre 2020. URL : http://journals.openedition.org/actesbranly/80; DOI : https://doi.org/10.4000/ actesbranly.80

Ce document a été généré automatiquement le 8 septembre 2020

(c) Tous droits réservés 


\title{
André Leroi-Gourhan et Pierre Francastel
}

\author{
Alain Schnapp et Pierre Lemonnier
}

Pierre Francastel et André Leroi-Gourhan ont vécu chacun une histoire dissemblable et nous ont laissé des œuvres très éloignées les unes des autres, mais quelque chose les rapproche. Ce sont des savants un peu à part qui ont une conscience vive de leur singularité; ils s'expriment tous deux avec retenue mais avec une puissante originalité ; ils n'occupent pas le pouvoir mais ils exercent leur influence en outsiders. Entre eux, onze ans de différence - 1900-1911 -, une période suffisante pour marquer les écarts, mais en même temps assez courte pour pouvoir les englober dans une même génération formée au contact de l'Année sociologique, de la fondation du musée de l'Homme et de la création des Annales.

2 Ce sont des outsiders : ni l'École normale, ni l'agrégation n'ont sanctionné les études de l'un ou de l'autre, ni l'un ni l'autre ne sont passés par les Écoles françaises à l'étranger, mais ils ont commencé leur vie professionnelle par des bourses, de la fondation Rockefeller pour Francastel à Versailles, du gouvernement japonais à Kyoto pour LeroiGourhan. Tous deux ont eu une expérience internationale, la Pologne fondatrice pour Francastel, le Japon pour Leroi-Gourhan. Tous deux ont atteint la reconnaissance après guerre à la VIe section de l'EPHE pour Francastel, à Lyon puis à la Sorbonne, et au Collège de France pour Leroi-Gourhan. Tous deux ont subi des échecs, à la Sorbonne pour Francastel qui se présenta en vain à la succession de Lavedan, au musée de l'Homme dont il souhaitait la direction pour Leroi-Gourhan. Tous deux ont été des chercheurs prolifiques, auteurs très jeunes de travaux qui attirent l'attention. La thèse d'État de Francastel sur la sculpture à Versailles est publiée en 1930 par les éditions Morancé, elle est accompagnée de son étude sur la naissance du musée historique de Versailles. Elle a été précédée par son étude sur Giraudon en 1928. Plus surprenante encore est la productivité d'André Leroi-Gourhan : en 1936, Bestiaire du bronze chinois et La civilisation du renne, en 1936 encore, L'Homme et la Nature. En 1943, les Documents pour l'art comparé de l'Eurasie septentrionale et, incroyable somme, L'Homme et la Matière, en 1945 Milieu et Techniques, en 1946 L'Archéologie du Pacifique Nord. On le voit, des œuvres 
importantes et précoces. Mais on relève d'emblée une différence. Francastel part d'un lieu bien déterminé, l'histoire de l'art, et il déploie sa curiosité sur des voies a priori classiques : Versailles, Giraudon, l'art du Moyen Âge. Sa production ne sort du champ convenu des études qu'après la guerre avec la thématique des lieux, de l'espace, de la perspective et des techniques. Leroi-Gourhan s'affirme dès le début en marge, dans un espace qu'il définit lui-même entre anthropologie, ethnologie et préhistoire. Il se veut à la fois à la fois généraliste et praticien d'un champ disciplinaire bien précis, homme de terrain et théoricien, observateur attentif des immenses espaces de l'Eurasie dont il possède les langues, et en même temps préhistorien qui développe une véritable éthique de la fouille à partir de son expérience tardive en tant que titulaire de la chaire d'ethnologie coloniale de l'université de Lyon. Bref, en 1945, l'œuvre de Leroi-Gourhan a déjà pris son pli, celle de Francastel s'épanouit. Il entre à cette époque dans une période d'intense créativité qui le conduit à définir le programme d'une nouvelle discipline, la sociologie de l'art.

Fig. 1. Pirogue de type masawa or tadob. Kitava, partie ouest du Kula ring (Massim province), Papouasie Nouvelle-Guinée, 1996

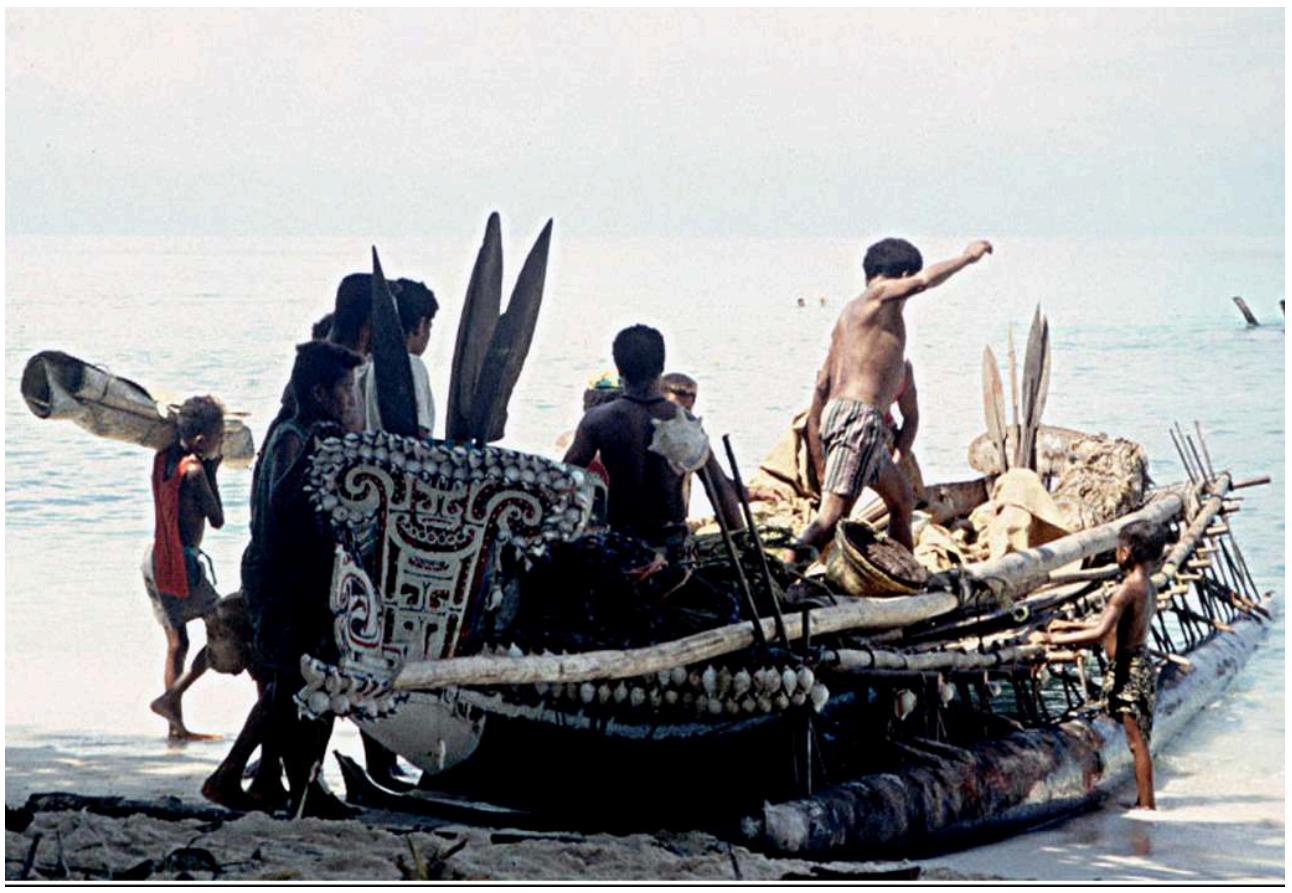

(c) Frederick Damon

On le voit, l'art des « vies parallèles " appliqué à Leroi-Gourhan et à Francastel révèle plus de dissemblances que de convergences. Pourtant leurs œuvres et leurs trajectoires, qui se sont plus côtoyées que rencontrées, dessinent une figure singulière de complémentarité, inscrivent un certain rapport entre l'art et les techniques dans un contexte original, celui du fait technique et de la chaîne opératoire maussienne. La création artistique est au cœur de leur approche. Ce questionnement recouvre celui d'une institution, comme le musée du quai Branly. Si on le considère dans la longue durée, le présent musée est un avatar au sens indien du terme du musée d'ethnographie du Trocadéro, du musée de l'Homme et du musée des Colonies, devenu musée des Arts africains et océaniens. Cette histoire compliquée, faite de déplacements, de transferts de collection et de changement de paradigme, est tout à fait singulière. 
Elle n'a pas d'équivalent ailleurs chez nos voisins, et elle s'inscrit dans le contexte particulier de l'art français qui, au tournant $\mathrm{du} \mathrm{xx}^{\mathrm{e}}$ siècle, est le laboratoire de la rencontre avec les arts dits primitifs. Certes, Franz Boas, en Amérique, s'était posé avant Francastel et Leroi-Gourhan la question du rapport entre l'art et les techniques; par ailleurs, la généalogie fonctionnelle des objets, imaginée par Pitt-Rivers à la fin du $\mathrm{XIX}^{\mathrm{e}}$ siècle, n'est pas sans relation avec la notion de type chez Leroi-Gourhan. La situation française est cependant déterminée par un climat particulier. Ce sont les artistes, les créateurs, qui posent aux ethnologues et aux archéologues les questionsclés. De leur côté, Marcel Griaule, Georges-Henri Rivière, Michel Leiris et, bien sûr, André Breton, ont leur part dans cette exceptionnelle aventure. Reste que Francastel et Leroi-Gourhan ont joué ici, chacun dans sa dissemblance, un rôle décisif et complémentaire.

\section{Deux œuvres convergentes}

4 Aux marges de l'histoire historienne qu'il néglige et parfois méconnaît, Leroi-Gourhan veut établir une continuité entre histoire de la nature et histoire des hommes en société. Son grand modèle est Cuvier : il a étudié en autodidacte de génie la zoologie et la paléontologie, et il puise sans se soucier des conséquences dans l'arsenal de la biologie descriptive d'un Paul Broca ou d'un Marcellin Boulle pour fonder les mécaniques qui sous-tendent son œuvre. Leroi-Gourhan s'est toujours méfié des sources écrites, des archives au sens scripturaire : comme un artiste, il compose son œuvre avec des échantillons acquis ou ramassés au gré de ses voyages ou de ses expéditions aux Puces, de dessins et de fiches compulsivement annotées dans chaque musée. Dès avant son voyage au Japon, il a construit une véritable Wunderkammer faite d'objets ou de croquis, de dossiers et d'un petit nombre de livres de référence, tous annotés et rangés avec un soin méticuleux. Pour son ouvrage consacré à l'art comparé de l'Asie septentrionale, il annonce modestement qu'il a amassé neuf mille illustrations, dessinées de sa main et classées. Cet art de la fiche se déploie ensuite dans Évolution et Techniques, synthèse originale qui entend créer un répertoire de l'ensemble des stratégies de l'espèce humaine à travers l'étude de milliers d'artefacts. Collectionneur invétéré, Leroi-Gourhan est un bourreau de travail qui fait de son atelier un laboratoire. Il sera toute sa vie un créateur infatigable d'équipes diversifiées, qui s'attache à importer dans le champ des sciences de l'Homme la dure éthique des protocoles et des classements systématiques.

5 On est moins bien renseigné sur les méthodes de Francastel, d'abord parce que l'approche de l'historien de l'art laisse par définition moins de traces que celle de l'ethnologue ou du préhistorien; ensuite du fait que Francastel est par vocation un homme de musée et par-dessus tout, à l'inverse de Leroi-Gourhan, un homme de cabinet. Cela ne signifie pas qu'il néglige ce qu'on appelle en ethnologie, le terrain. Pour lui la technique, le métier, l'observation minutieuse des stratégies et des techniques d'exécution sont le point de départ. Qu'il s'agisse de l'étude sur Versailles ou de la monographie d'artiste, la même attention aux œuvres est patente :

«Il est absolument faux de penser que les œuvres d'art, qu'il s'agisse d'ailleurs de monument ou d'œuvres figuratives, aient une réalité et puissent être créées indépendamment de la collaboration d'un artiste créateur et d'un certain cercle de témoins. L'œuvre d'art n'est pas un objet naturel de plus à rajouter à la 
nomenclature du créateur. Elle est un lieu de rencontre entre des esprits, elle est signe. ${ }^{1}$ " Gourhan dans ses Documents pour l'art comparé de l'Eurasie :

«Il est certain que les formes les plus anciennes et les plus rustiques connues de l'art sont déjà le fruit d'une longue tradition et que l'on ne peut à aucun degré admettre que les Africains ou les Polynésiens représentent l'état de naïveté d'un début. ${ }^{2}$ "

7 Sous des formes différentes, les deux auteurs mettent au cœur de leur approche de l'art la sociabilité qui le définit. Francastel insiste sur la relation qui lie l'artiste à son milieu, Leroi-Gourhan attire l'attention sur la vacuité de l'idée de primitivisme appliquée à l'art des sociétés non occidentales. Tous deux viennent d'horizons différents, leurs objectifs ne sont pas les mêmes, mais ils se retrouvent fondamentalement dans une définition maussienne du rôle de la technique et de la sociabilité dans le travail artistique. En examinant leur vision du champ artistique et de la chaîne opératoire, nous espérons éclairer une part d'une question centrale pour l'étude des arts non européens ou même des arts dits mineurs. Toutes les cultures produisent des objets utiles et nécessaires à la vie collective, mais les sociétés préindustrielles, parce qu'elles ont précisément recours à une chaîne de production artisanale, n'opèrent pas de distinction entre un type de travail qui vise à fabriquer des commodités et un autre qui élabore ce que nous appelons des œuvres d'art. Pierre-Maxime Schuhl et Jean-Pierre Vernant ont bien montré que technè, en grec, conjoint les deux pratiques, et que le mot « art », dans le sens qu'il a depuis la Renaissance, n'aurait pas été intelligible pour un Grec de l'époque classique. De même, il a fallu tout le travail philologique de Marcel Detienne et Vernant pour récupérer l'ancienne notion de mètis, l'intelligence rusée, qui n'évoque rien pour nous. La mise en évidence des catégories de la production artistique est au cœur de la réflexion de nos deux auteurs; elle est liée à la question posée par Ignace Meyerson sur les rapports entre les fonctions et les œuvres. Francastel avait beaucoup lu Meyerson et réciproquement.

8 Pour ce qui est de Leroi-Gourhan, sa pratique, dans les synthèses, de réduire la bibliographie au strict nécessaire, ne permet pas de reconstituer facilement ses sources. L'attention qu'il porte à l'outil, la volonté de construire un système général des objets l'apparentent cependant par ses voies propres à l'approche de Meyerson. Le fameux tableau consacré à l'évolution du tranchant des silex, quelles que soient les modifications qu'on peut lui apporter aujourd'hui, renvoie à ce type de questionnement. Une différence est cependant patente : là où Francastel, en maussien rigoureux, cherche à déterminer les contraintes sociales qui conditionnent le travail artisanal ou artistique, Leroi-Gourhan s'emploie à reconnaître des lois générales qui inscrivent la production des objets dans un processus induit par les principes de l'évolution. Francastel donne cette définition de l'évolution dans Arts et techniques:

«Il va de soi que les plus grandes transformations survenues dans l'outillage matériel et intellectuel de l'homme n'ont jamais d'un seul coup transformé sa destinée. On a insisté plus haut sur l'importance de la distinction nécessaire entre le fait de la découverte et celui de son adaptation, conformément aux belles observations de Leroi-Gourhan. ${ }^{3}$ "

Il s'appuie sur Leroi-Gourhan pour mieux définir la notion maussienne d'outillage et pour rappeler le caractère aléatoire des inventions, qui ne débouchent sur un progrès que lorsqu'elles sont intégrées dans une chaîne opératoire. Cela lui permet de mieux 
cerner la polysémie de la notion d'art : «Par conséquent l'œuvre d'art est le produit unique d'une activité qui se situe à la fois sur le plan des activités matérielles et des activités imaginaires d'un groupe social donné. Dans les deux cas, au surplus, elle possède un double caractère sociologique et individuel au même titre que la personnalité de l'homme qui l'a produite. ${ }^{4}$ »

Dans sa préface à Fil du temps, qui est une sorte de bilan de son œuvre, en 1983, LeroiGourhan s'explique ainsi sur sa conception de la mentalité des hommes de la préhistoire :

« Nous ne savons pas encore très bien à partir de quoi se sont développées ces activités esthétiques, religieuses, ludiques, car les témoins ne sont pas en état d'être interprétés. En effet, on est loin encore d'avoir une prise solide sur la musique, la décoration corporelle, la magie, le jeu, toutes formes d'activité qui relèvent du même ensemble étroitement lié au développement du langage. La recherche des premiers hommes, et dans ces hommes celle du capital intellectuel, m'a vu emprunter les différentes voies qui sont offertes, aboutissant chaque fois à une vision différente de l'homme en question. ${ }^{5}$ »

11 Entre la position de Francastel et celle de Leroi-Gourhan, beaucoup de points communs, l'idée d'outillage, de capital intellectuel, l'intérêt pour le matériel et l'imaginaire, mais Francastel est plutôt polaire tandis que Leroi-Gourhan préfère l'image du spectre, " esthétique, religieux, ludique ». Ce dernier insiste surtout sur les " voies différentes », qui permettent de saisir l'activité artistique. Il se trouve que les deux hommes sont également musiciens, Francastel étant un passionné de musique classique, LeroiGourhan d'une musique vernaculaire qui va du biniou au violon tsigane.

Reste quand même une différence majeure : Leroi-Gourhan, l'auteur de L'homme et la Matière, se veut «technomorphologue »; le mot et la méthode associée figurent dans son travail publié par l'Encyclopédie française permanente dès 1935. Cela signifie pour lui une continuité entre le naturel et le culturel, l'environnement et la société, qu'il exprime ainsi :

« Tout semble se passer comme si un prototype idéal de poisson ou de silex taillé se développait selon des lignes préconcevables, du poisson à l'amphibien, au reptile, au mammifère ou à l'oiseau, du silex indifférencié dans sa forme au tranchet de pierre polie, au couteau de cuivre, au sabre d'acier. Qu'on ne s'y trompe pas, ces lignes rendent simplement un aspect de la vie, celui du choix inévitable et limité que le milieu propose à la matière vivante... Le déterminisme technique est aussi marqué que celui de la technologie. ${ }^{6}$ "

13 Le Leroi-Gourhan de 1943 est différent de celui de 1983, mais il continue de se situer dans un schéma évolutionniste que Francastel récuse. La part de nécessité et d'invention qui préside à tout progrès technique n'est pas gouvernée, pour ce dernier, par un modèle déterministe :

"Quelle est dans la société actuelle, la part réciproque des outils, des instruments et des machines? Suivant quel rythme historique s'est faite la substitution toujours plus grande des objets du dernier type à ceux du premier? Quelles furent par exemple les étapes de la substitution générale des procédés de soudure, de rivetage ou de brasage aux méthodes de l'assemblage, et avec quelles répercussions sur la main-d'œuvre et le goût ? ${ }^{7}$ "

14 Le vocabulaire est le même, mais Francastel historicise des catégories qui tendent chez Leroi-Gourhan à apparaître comme naturelles. Reste qu'on peut déceler malgré ces divergences des points de rencontre. 
Leroi-Gourhan entend dès ses premiers articles classer l'ensemble des objets et artefacts produits par l'humanité en un système descriptif global qui devient une encyclopédie d'histoire de la nature et des techniques. Le projet s'énonce comme universel avec un sous-titre un peu oublié : Évolution et technique; le tome I s'intitule: L'Homme et la Matière, le tome II : Milieu et Technique. La définition du projet est plus qu'ambitieuse, il s'agit de faire parler les objets pour les constituer en système :

« Dans le tableau immense de la vie des hommes de tous les temps que l'Ethnologie tend à peindre, les techniques jouissent d'une place singulièrement importante. C'est par elle que nous dépassons la marge très étroite des textes et des rapports oraux. À partir du point ou l'homme ne peut plus parler parce qu'il est absent ou mort, où les archives font défaut, des témoignages subsistent : celui de l'art et celui des techniques. ${ }^{8} "$

La position de Leroi-Gourhan est épistémologique; l'ethnologue doit inventer une technique capable de suppléer à l'absence de textes ou de discours. Ce qui justifie le projet, c'est précisément cette capacité de l'ethnologue de faire parler ce qui est muet. Dans la perspective qui est la nôtre, la distinction opérée entre l'art et les techniques n'est pas sans intérêt sauf que la différence entre l'un et l'autre domaine n'est pas donnée... Dans le travail de Leroi-Gourhan, des études d'œuvres d'art, de Bestiaire du bronze chinois à Préhistoire de l'art occidental, jouent un rôle important qui est parallèle à ses études de technologie. Pourtant, s'il s'emploie avec constance à définir ce qu'il entend par technologie à travers la tendance et le fait, il ne donne pas de définition substantive de ce qu'il entend par art. La notion est pourtant là, en creux, dès les Documents pour l'art comparé de l'Eurasie septentrionale. On a vu que son approche ici, comme plus tard pour les grottes ornées, est sérielle, qu'elle cherche à prendre en compte des ensembles et non des œuvres isolées. Les objets sont définis comme des documents qui sont extraits de tous les types de support possibles: objets sculptés, peints, gravés, tissus brodés. Pour établir cet ensemble énorme, tant géographique que chronologique, Leroi-Gourhan part d'une hypothèse :

«On peut admettre pourtant comme une donnée de bon sens que le réalisme ou l'intention de réalisme soient à l'origine même de la figuration; leur conservation étant constamment compromise par la simplification, la recherche décorative ou les contraintes matérielles $»^{9}$.

Mais le réalisme ainsi invoqué qui joue un rôle si déterminant dans l'art pariétal est aussi nuancé par son contraire :

"Ainsi considérant le réalisme comme naturel, nous admettons immédiatement l'existence d'une réalité non moins puissante : la stylisation. Réalisme et stylisation apparaissent alors comme deux aspects d'une seule source : la tendance figurative, et c'est par un effet rétrospectif que nous dissocions dans une œuvre ce qu'elle a de réaliste ou de stylisé, comme si, par un effort conscient, l'époque imposait aux doigts humains le dosage..$^{10} »$

18 La proximité avec Francastel est ici évidente; dans la suite du texte déjà cité de Sociologie de l'art, ce dernier écrit ceci qui apparaît comme un commentaire mesuré des intuitions de Leroi-Gourhan :

«Il existe, en effet une communauté de nature entre les différentes formes d'activité de l'esprit humain, qui dans son essence est un, et il ne saurait être question d'opposer par conséquent une connaissance figurative à la connaissance intellectuelle, mathématique, verbale ou expérimentale... À la base de chacune des activités spécifiques de l'esprit, l'opératoire l'emporte, rendant possible l'exploration d'un réseau de sensation et d'expériences limites. ${ }^{11}{ }^{\prime}$ 
Chez Leroi-Gourhan, le modèle naturaliste reste la référence, il informe sa théorie comme sa pratique d'ethnologue et de préhistorien; chez Francastel l'influence des Annales et de l'école sociologique est plus prégnante. Ils se rejoignent cependant dans leur volonté de mettre les œuvres en système et de questionner le processus de création dans sa dimension sociale. Pour eux les artefacts sont le produit d'une négociation complexe entre les impératifs de la matière et les attentes de la société.

\section{Dialogues imaginaires sur les techniques et l'esthétique}

Passionnés par la matérialité des choses, attentifs aux techniques et aux objets simplement utiles, Leroi-Gourhan et Francastel ont refusé l'opposition entre technique et esthétique, entre objet modeste et œuvre d'art. Ils ont au contraire exploré les rapports entre technique et esthétique, et la façon dont les hommes organisent ces rapports pour vivre en société et produire de la société, en soulignant que «les techniques dominent tous les arts, y compris la peinture dans ses formes les plus libres $»^{12}$, ou encore que " l'art est lui-même, dans une certaine mesure, une technique sous le double plan des activités opératoires et figuratives ${ }^{13}$. On ne peut comprendre l'art sans saisir ses liens matériels et mentaux avec la culture matérielle (ou le système technique) du moment. Quel que soit ce moment, depuis la Préhistoire jusqu'à la révolution industrielle, où les « relations traditionnelles entre le beau et l'utile ou entre art et technique » n'ont pas été modifiées ${ }^{14}$. Plus largement, chacun a voulu les replacer dans le contexte global des sociétés et de cultures qui les créent, transforment ou rencontrent, pour comprendre ce qui a pu déterminer leur état autant que leur rôle social du moment - ce qui revient au même.

21 Évoquer la volonté de ces deux auteurs de penser simultanément l'art et la technique, c'est d'abord se tourner vers une époque de curiosité et d'effervescence intellectuelle, celle des Annales, déjà mentionnées, où ces diverses disciplines, souvent, dialoguaient. Un seul exemple : écrite par Lucien Febvre dans un ouvrage édité par Leroi-Gourhan dans lequel Francastel publia également la phrase qui suit pourrait aussi bien être de Bertrand Gille :

«Non, l'activité technique ne saurait s'isoler des autres activités humaines.

Fortement encadrée par celles-ci - qu'il s'agisse de religion, d'art ou de philosophie,

de besoins économiques ou de besoins militaires - elle est commandée par l'action

combinée de tous ces facteurs convergents... $»^{15}$.

Comme souligné à l'instant, lorsque Francastel propose une version déterministe de $l^{\prime}$ histoire des techniques et ses rapports avec l'art des $\mathrm{XIX}^{\mathrm{e}}$ et $\mathrm{xx}^{\mathrm{e}}$ siècles ${ }^{16}$, il aborde à sa manière les questions qui sont aussi celles de Bertrand Gille, de Leroi-Gourhan ou d'André Varagnac. Et, à propos, encore, de Bertrand Gille, ce spécialiste de l'industrie du fer (1947), des ingénieurs de la Renaissance (1964) et des mécaniciens grecs (1980) qui rédigèrent à la fin de sa vie (1978) une fresque mêlant histoire, économie et sociologie autour de la notion de "système technique ": qu'a-t-il bien pu dire à Francastel, ce jour de 1952 ou 1953 où ils participaient ensemble au séminaire du père de la «psychologie historique », Ignace Meyerson ${ }^{17}$ ? 
Fig. 2. Ignames Maabutap décorées. Cérémonie Waapi Saaki, Nyamikum (East Sepik Province), Papouasie Nouvelle-Guinée, 2003

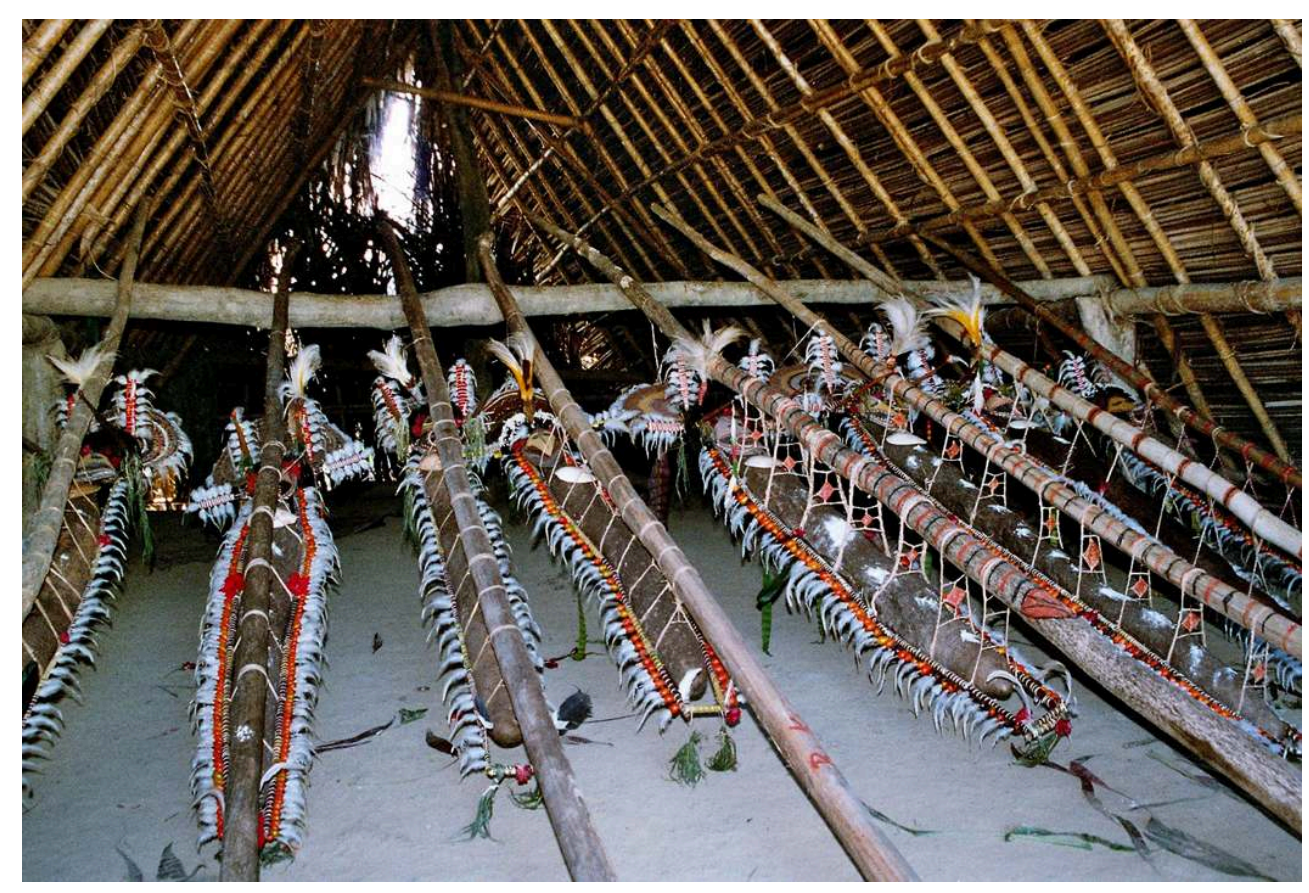

(c) Ludovic Coupaye

Rapprocher ces deux auteurs, c'est aussi retrouver les fondements méthodologiques des enquêtes et des analyses qui ont permis d'entrer de plain-pied dans les recherches associant systématiquement l'étude des techniques à celle de l'esthétique, qu'ils avaient imaginées dans le prolongement direct de la pensée de Marcel Mauss. Pour le chercheur de ce début de xxi ${ }^{e}$ siècle, lire l'ethnologue-préhistorien ou le sociologue de l'art est un régal, au point que des formules longues de seulement deux lignes résonnent encore comme des programmes pour le présent. Lorsqu'il évoque : «C'est dans l'adhérence à l'efficacité pratique ou spirituelle de l'acte humain socialisé que résident la force et les prestiges des grandes œuvres $»^{18}$, Francastel n'est pas loin du programme d'Alfred Gell sur l'agency des objets ([1998] 2009), d'ailleurs lui-même déjà en germe chez Mauss ${ }^{19}$.

Au-delà de leur influence réciproque et de leur filiation commune avec Mauss, Francastel et Leroi-Gourhan ont abordé des questions qui restent d'actualité au moment où, plus que jamais, l'histoire, la sociologie, l'anthropologie, la philosophie et l'ethnologie des techniques tentent de se pencher ensemble sur le rôle des objets dans les cultures et les organisations sociales ${ }^{20}$. Pourtant, leur approche novatrice portait également en elle une limitation que leurs successeurs n'ont que récemment dépassée... en suivant les méthodes qu'ils prônaient.

\section{Retour sur l'héritage de Mauss}

Pour qui constate la séparation radicale effectuée aujourd'hui entre les objets exposés pour leurs qualités esthétiques dans les collections permanentes d'un musée comme celui du quai Branly, et ceux qui sont supposés strictement utilitaires, Francastel et Leroi-Gourhan sont d'abord remarquables par leur refus partagé de toute coupure 
entre technique et art. L'un comme l'autre ont considéré ces deux domaines de l'activité humaine comme des productions sociales à part entière. Tous deux affirmaient également qu'art et technique ont, dans la condition des hommes d'un lieu et d'un temps déterminés, un rôle qui n'est ni seulement technique (entendre ici, physiquement opératoire) ni seulement esthétique (quoi que l'on entende par là), mais bien plus vaste. Leroi-Gourhan déclarait ainsi avoir voulu élucider par palier « tout ce qui adhère aux raisons passant par la matière et la technique $»^{21}$. Et, comme Francastel, il pensait que les modalités sociologiques et psychologiques de l'insertion de la technique et de l'art dans les systèmes culturels doivent être étudiées de façon comparée et en de l'Ethnologie générale (1968), le sociologue de l'art ne dit pas autre chose, même s'il n'y cite plus Leroi-Gourhan, auquel il avait régulièrement fait référence, et sur des thèmes semblables, dans son article "Technique et Esthétique " ([1948] 1953) et ou dans Art et technique au XIX et XXe siècles (1956).

Comme on l'a rappelé plus haut, cette prise en considération conjointe de l'art et de la technique leur venait de Mauss, écrivant par exemple dans le Manuel d'ethnographie, qu'

«il est très difficile de distinguer les phénomènes esthétiques des phénomènes techniques pour une raison précise : une technique est toujours une série d'actes traditionnels ; une série, c'est-à-dire un enchaînement organique destiné à produire un effet qui n'est pas seulement un effet sui generis, comme dans la religion, mais un effet physique. Or, très souvent", poursuivait leur maître commun, "l'œuvre esthétique consiste en un objet. La distinction entre les techniques et les arts, surtout lorsqu'il s'agit d'arts créateurs, n'est donc qu'une distinction de psychologie collective: dans un cas, l'objet a été fabriqué et pensé par rapport à son but physique ; dans l'autre cas, il a été pensé par rapport à la recherche de la sensation esthétique. L'étranger connaîtra la distinction en interrogeant d'abord l'auteur ou l'acteur. Il y a de la technique dans l'art et il y a une architecture technique; mais l'objet esthétique se reconnaît à la présence d'une notion plus compliquée que la notion d'utilité. ${ }^{22}$ »

Enfin, tous deux ont mis l'universalité de la fonction esthétique au cœur de leurs préoccupations. Non pas l'universalité du «beau», idée à laquelle le jeune LeroiGourhan adhérait, mais celle de la fonction esthétique. Pour Francastel, «l'art est une des fonctions permanentes de l'homme, la fonction esthétique est universelle, le beau est relatif $\aleph^{23}$.

Pour Leroi-Gourhan, l'esthétique est ce qui, pour une large part, donne leur saveur locale aux productions humaines, y compris aux objets dont la fonction première est l'action sur la matière. Sans citer Boas sur l'idée que l'art est rythme ${ }^{24}$ - ni Mauss ${ }^{25}$, d'ailleurs, qui lui aussi associait les rythmes et les équilibres à l'esthétique en citant Boas - Leroi-Gourhan écrit par exemple :

«Le pithécanthrope n'aurait pas pu satisfaire des besoins s'il n'avait eu le désir de faire un outil ; il n'aurait pas pu le faire non plus - et encore moins - s'il n'avait obtenu que cet outil fut équilibré, symétrique, bien fait [...]. On ne comprend pas pourquoi ils se donnaient tant de mal à tailler de beaux outils alors que la plupart du temps un bon éclat tiré d'un caillou permet d'avoir un tranchant qui ne vous coupe pas la main mais débite fort bien ce que l'on veut tailler [...]. Cette régularité, ce surcroît d'esthétique sur des objets qui devaient être tristement utilitaires, cela aurait-il pu susciter s'ils n'avaient pas marqué le désir de faire quelque chose de bien. ${ }^{26}$ »(souligné par nous).

De Mauss, Francastel et Leroi-Gourhan tenaient également leur intérêt pour les objets "ordinaires", usuels, ceux qui ne nous frappent pas par leur fonction esthétique, comme nous l'avons signalé, et ce faisant, ils partageaient les motivations et l'ambition 
« utopique » de Mauss : tout collecter, interroger largement, documenter, comprendre la mécanique des objets, saisir les jugements locaux ${ }^{27}$. Ces pionniers rappellent donc à ceux qui en douteraient que l'on ne peut comprendre une œuvre d'art sans se pencher tout autant sur les objets modestes. Y compris pour aborder ces questions nouvelles que soulèvent, par exemple, le rôle sui generis des objets dans l'action rituelle ou l'inscription d'une préoccupation « esthétique » dans la structure même des objets, et pas dans leur décor.

Leurs positions identiques sur l'art et la technique n'impliquent pas que les deux hommes aient échangé à ce propos. Leroi-Gourhan ne dit pas grand-chose de Francastel : « ... pas follement sympathique, mais follement intelligent $»^{28}$. Francastel, en revanche, cite et reprend l'auteur de Milieu et technique ${ }^{29}$ à propos de "l'adaptation et [... de] l'insertion pratique d'une technique ou d'un outil dans des formes historiques de l'activité humaine $»^{30}$, c'est-à-dire, lorsqu'il s'interroge sur l'emprunt et l'invention, bref sur les déterminants multiples des choix techniques ${ }^{31}$ et sur leurs motivations non techniques, elles aussi déjà soulignées par Mauss ${ }^{32}$. Du chapitre de Leroi-Gourhan sur la "tendance technique», qu'il juge "remarquable», Francastel a retenu que l'appareillage de description, de comparaison et d'analyse présenté dans Milieu et Technique permet de rendre comprendre en quoi « l'adaptation d'un objet à la vie d'une époque et d'un groupe social donnés va au-delà de la pure technique $»^{33}$. Quarante ans plus tard, Francastel ne cite plus explicitement Leroi-Gourhan ${ }^{34}$, mais la référence est évidente.

31 En vérité, la référence au binôme tendance-fait utilisé par Leroi-Gourhan pour construire puis compulser la gigantesque classification des techniques proposée dans Évolution et techniques ${ }^{35}$ se situe au cœur de la problématique des deux auteurs, à savoir : comprendre, à travers toutes sortes d'objets, les rapports entre technique et esthétique, ainsi - et surtout - que la manière dont les hommes organisent ces rapports pour vivre en société et produire de la société. Rappelons que, chez Leroi-Gourhan, ce binôme donne un cadre d'analyse et un début d'explication au dilemme que représente l'engendrement par des tendances universelles de techniques et d'objets qui sont pourtant propres à un peuple, à un lieu et à un temps donnés. D'un côté, les techniques expriment partout simultanément des lois de la matière et une propension humaine à l'économie d'effort qui limitent l'éventail des options retenues. Mais, d'un autre côté, elles sont pétries de significations et de logiques qui ne sont pas d'abord concernées par l'action sur la matière.

Par «tendance ", Leroi-Gourhan a désigné à la fois la propension qu'ont les humains à effectuer une action donnée (couper des arbres) et leur recours aux moyens les plus adaptés pour effectuer cette action (écraser les fibres du bois avec un tranchant frappant le tronc à la volée, par exemple), compte tenu des contraintes physiques qui pèsent sur elle. Ainsi, en tout temps et tout lieu, la «tendance » est-elle de couper des arbres, mais aussi d'utiliser des outils du type herminette, dont l'organisation et la dynamique sont les plus propices au coupage du bois en développant le moins d'effort possible. La tendance ne traduit donc pas autre chose que le déterminisme de la matière et une sorte de course au progrès, en l'occurrence le désir d'augmenter l'impact d'un outil de pierre en le dotant d'un manche - ce qui permet également de ne pas s'écraser les doigts!

On a déjà noté que son excellence dans cet « art de la fiche » a permis à Leroi-Gourhan de rendre compte des caractéristiques mécaniques de quelque 45000 des objets du 
musée de l'Homme (désormais versés aux collections du musée du quai Branly). C'est à partir de ce corpus comparatif qu'il a indiqué à la fois les actions élémentaires sur la matière autour desquelles diverses familles d'objets s'organisent et la façon dont elles cristallisent dans la culture matérielle de chaque groupe humain et de chaque époque sous la forme de «faits" observables, c'est-à-dire des outils et des variantes des procédés techniques auxquels ils répondent.

Bien que le programme de classification et d'analyse des objets développé par l'ethnologue devenu préhistorien et admiré par Francastel répondît très exactement au vaste projet dans lequel Mauss considérait les techniques et les arts comme des productions sociales susceptibles de varier au gré des cultures, il y a lieu de noter que, selon ses propres termes, Leroi-Gourhan s'éloignait de son maître sur deux points. D'abord, il considérait que, influencé par les penseurs allemands et les Kulturkreise (" cercles culturels » de diffusion d'un trait culturel), Mauss prêtait trop d'importance à la question des origines (des techniques, des styles), alors que lui-même cherchait à exposer et à comprendre la variabilité des objets - en quoi celui-ci diffère-t-il de celuilà, et pourquoi ? -, sans privilégier leur origine, mais dans le but de saisir sur quoi porte leur différence, à la fois fonctionnellement (mécaniquement, si l'on veut) et par leurs usages et représentations.

Leroi-Gourhan reprochait également à Mauss son manque de " métier ", notamment un manque de connaissances mécaniques et d'expérience des choses en fonctionnement sans lequel il n'est pas possible de repérer les «moyens élémentaires d'action sur la matière " à partir desquels établir une comparaison systématique des objets (et des techniques). Notons au passage que ce «métier» - sans lequel toute analyse ethnologique des techniques relève de l'amateurisme - était ce que Lévi-Strauss soulignait dans son hommage à Leroi-Gourhan :

«Et ce qui rend, entre autres, son apport irremplaçable, c'est d'avoir enseigné à ses étudiants, c'est de nous avoir enseigné à tous, qu'il est impossible de parler de quoi que ce soit sans savoir d'abord de quoi on parle. [...] Rien mieux qu'un tel précepte ne peut nous mettre à l'abri de ces tentations dont nous sentons aujourd'hui le danger: d'une sorte de verbiage sociologique ou sémiotique qui croit possible de parler de la symbolique ou de la fonction significative de tels ou tels objets matériels sans s'être d'abord assuré de ce qu'ils sont en eux-mêmes et comment ils ont été fabriqués. ${ }^{36}{ }^{\prime}$

C'est pourtant bien le programme de Mauss, y compris tout ce qui, chez ce dernier, évoque déjà l'usage que l'on fera de la notion de "chaîne opératoire " ${ }^{37}$, que LeroiGourhan avait en tête en réalisant les fiches dont l'analyse constitue le "terrain » sousjacent aux deux volumes d'Évolution et techniques dans lesquels il compare les techniques, c'est-à-dire classe logiquement les objets (outils, armes, contenants...) en fonction des moyens d'action sur la matière que leur fabrication ou leur usage mettent en œuvre ${ }^{38}$. Car, par définition, l'ethnologie consiste à rendre compte de ce que l'on a sous les yeux, ici et maintenant, et à souligner en quoi un discours, une institution, une pensée partagée, un objet, une technique, etc. sont propres au groupe étudié. Classer les objets, c'est se donner les moyens de localiser et d'expliciter ces différences et ces ressemblances. On sait que Leroi-Gourhan a suffisamment convaincu ses collègues et étudiants pour que des ethnologues et des préhistoriens, par dizaines, recueillent et analysent ces "chaînes opératoires", la donnée de base d'une ethnologie des techniques étant inscrite dès l'origine dans une recherche sur l'esthétique. Quant à Francastel, il a utilisé le travail de Leroi-Gourhan et celui de Mauss pour donner une 
base à ses observations sur le métier et la technique des artistes et pour intégrer l'histoire de l'art dans une histoire générale des sociétés qui répond fondamentalement au programme des Annales. Pourtant, c'est seulement depuis une quinzaine d'années, et en ethnologie du lointain, qu'après bien des détours l'étude conjointe des arts et des techniques voulue par nos deux savants a commencé de se réaliser. Paradoxalement, il fallait d'abord se dégager de l'une des plus anciennes notions communes à l'archéologie, à l'ethnologie et à l'histoire de l'art : celle de style.

\section{L'obsession du style}

37 Le rapprochement de l'ethnologie des techniques et de l'anthropologie (ou de la sociologie) de l'art n'a pourtant pas eu lieu. C'est seulement aujourd'hui que ce rapprochement porte tous ses fruits, notamment grâce aux développements récents de la «technologie culturelle », discipline fondée par Leroi-Gourhan et développée autour de ses élèves Hélène Balfet et Robert Cresswell.Du programme commun à Francastel et Leroi-Gourhan, celui qui niait toute solution de continuité entre arts et techniques, tous les éléments demeurent, mais le contexte scientifique dans lequel il s'exerce désormais a radicalement changé. Comme on le verra, parallèlement au «retour à l'objet » dans l'anthropologie des années 1990 et à un intérêt pour ses «carrières" ou pour sa «vie sociale $\aleph^{39}$ qui ne firent aucun cas de la fabrication matérielle et de l'usage physique choses, les trouvailles s'accumulent désormais, qui saisissent comment l'art (et les techniques) est «à cheval sur trois niveaux distincts de nos activités mentales: le niveau technique, le niveau fonctionnel et le niveau significatif $»^{40}$.

Pour que ce rapprochement entre la technologie culturelle et l'anthropologie de l'art eût lieu, il fallait que s'effectue un déplacement des questions communes à Francastel et Leroi-Gourhan sur le style. D'abord, l'ethnographie menée avec les méthodes de Leroi-Gourhan et dans l'esprit de Mauss ${ }^{41}$, a conduit les études de technologie culturelle à porter notre regard et modifié notre compréhension des phénomènes sur lesquels s'exercent les choix «culturels », de «style ethnique » concernant les objets, c'est-àdire, des motivations «non techniques » des modes d'action sur la matière. Ce sont les matériaux patiemment recueillis avec le niveau de détail voulu par Leroi-Gourhan, et en portant attention aux interrelations des techniques, tant dans une même société qu'entre sociétés, comme lui-même ou Francastel le préconisaient, qui ont conduit à remettre en cause la vision stylistique des rapports entre art, technique et culture.

Comme on l'a dit, le déterminisme des lois de la nature était tel, pour Leroi-Gourhan, que les "premiers degrés du fait " - si l'on préfère, les aspects les plus directement concernés par un mode très général d'action sur la matière - ne traduisaient que l'adéquation nécessaire de la forme d'un outil à sa fonction première, agir sur la matière. C'est pourquoi, à ce niveau de généralité, les outils (et les techniques les mettant en œuvre) sont, le plus souvent, aussi parfaits qu'ils pourraient l'être. Ainsi, partout sur terre, et après quelques (dizaines?) de millénaires, les herminettes de pierre de forme ou d'assemblage aberrants n'ont plus cours. Selon le préhistorien, étant donné le carcan que constitue la tendance, l'imagination des hommes se serait limitée à des aspects secondaires de l'action sur la matière. C'est là que se repère le "style ethnique » qui " pourrait [...] se définir comme la manière propre à une collectivité d'assumer et de marquer les formes, les valeurs et les rythmes $»^{42}$. Ici joue un autre déterminisme, qui prend la forme d'une capacité innée des humains à percevoir les 
rythmes et les valeurs ${ }^{43}$. C'est pour cette raison que lui-même n'a repéré la variété des solutions techniques développées par diverses sociétés que sur les branches extrêmes et les plus détaillées des arbres classificatoires, lieu où ressortaient le mieux les différences et les similarités des objets et des actions sur la matière qui leur est associée. Un peu comme si on avait mis seulement là les «signaux coûteux » dont parle Schaeffer ${ }^{44}$.

En d'autres termes, et toujours selon Leroi-Gourhan, la diversité ethnique ne s'exprimerait que dans des traits secondaires des objets (et, par extension, des procédés techniques), n'intervenant que peu ou pas du tout dans l'action sur la matière, bref, relevant de leur style (et de la communication) davantage que de leur fonction physique. Et c'est là, dans ces aspects matériels des objets qui sont physiquement inutiles, que se niche l'esthétique.

On peut faire l'hypothèse que cette limitation de la création esthétique et de l'inventivité des cultures à des variantes sans effets matériels a enrayé le programme de recherche par lequel tant Leroi-Gourhan que Francastel désiraient souligner l'imbrication des manières dont les hommes ont simultanément manipulé, en les pensant ensemble, les déterminants matériels et sociaux de l'art et des techniques. À défaut de proposer une histoire de ce détour de la recherche qui n'est lui-même qu'un avatar d'une conception archéologique ${ }^{45}$ de la culture et des relations sociales obnubilée par la notion d'identité - identité produite par l'archéologue pour rassembler des restes matériels d'une société, ou identité supposée concentrer les pensées et les actions de ceux qui les produisent -, on se propose de montrer que c'est en suivant les préceptes méthodologiques de Leroi-Gourhan que s'effectue désormais le rapprochement, ô combien productif, entre la technologie culturelle et l'anthropologie l'art.

\section{Quand arts et techniques se confondent dans l'anthropologie de terrain}

Sur ce point, on a beaucoup changé d'avis. On sait aujourd'hui que les choix culturels portent aussi sur ces "premiers du fait ", naguère supposés être à ce point déterminés par les lois de la matière et la soif d'efficacité de nos congénères que Leroi-Gourhan considérait qu'ils ne sauraient varier d'une société à l'autre. Ces "choix» - les guillemets signalent qu'il s'agit ici de logiques non intentionnelles, et non de décisions individuelles ou collectives - sont souvent lourds de conséquences ${ }^{46}$. Ils portent aussi bien sur des procédés plus efficaces que d'autres que sur des éléments de la chaîne opératoire qui sont parfaitement invisibles dans l'objet produit, par exemple dans ces poteries indiennes dont la fabrication a été étudiée par Marie-Claude Mahias ${ }^{47}$. Ici, le choix concerne le recours à du crottin d'âne plutôt que de cheval comme dégraissant. Ni l'utilisateur ni l'observateur ne peuvent repérer dans l'objet l'option retenue lors des premières étapes de sa fabrication, mais, pour ceux qui l'ont fabriqué, c'est tout l'équilibre du cosmos qui serait mis en cause si l'on avait recours à une des matières plutôt qu'à l'autre! Point essentiel, une telle relation entre un objet et des manières de penser ne peut être mise au jour qu'au terme d'une fine enquête de technologie culturelle portant sur la fabrication des poteries. Pour aboutir à ce résultat anthropologique, il fallait observer, décrire, transcrire et analyser des chaînes opératoires, se pencher sur le «faire», et pas seulement sur l'information 
éventuellement déchiffrable sur l'objet fini. Il fallait aussi s'intéresser à autre chose qu'à ce qui fascine habituellement les anthropologues, à savoir les relations sociales dans lesquelles les objets interviennent, notamment celles, sacro-saintes, qui organisent l'échange ou affichent des identités.

Fig. 3. Bol apira qagora sur une plateforme cérémonielle.

Paiement d'une compensation matrimoniale, Aorigi, îles Salomon,

1997

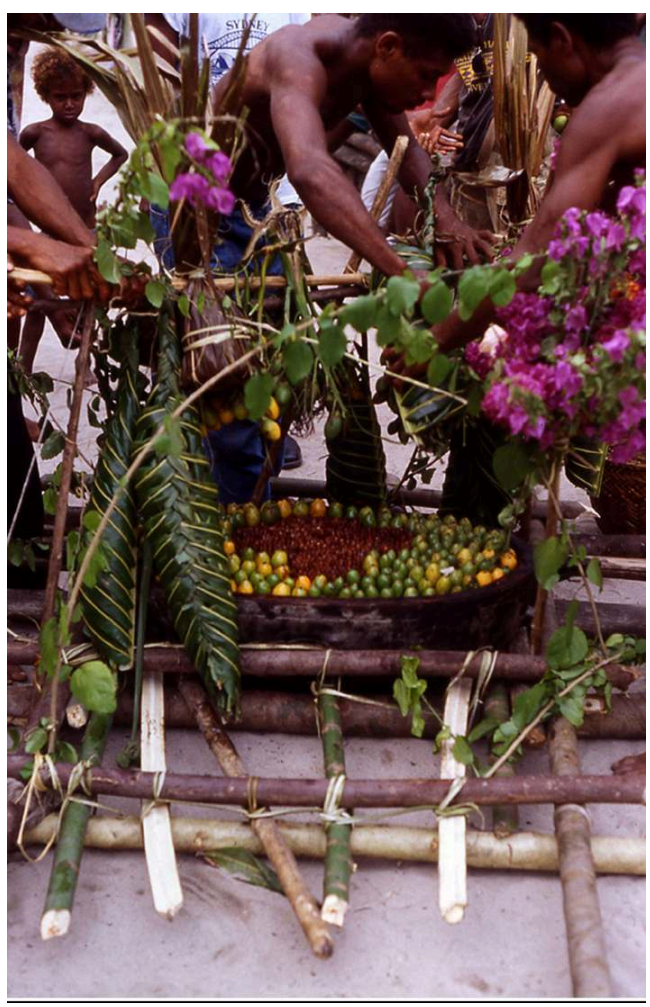

(c) Sandra Revolon

43 Nous savons désormais que les rapports que les groupes et les individus entretiennent avec des objets ne passent pas seulement par des sortes de messages inscrits sur ceux-ci, qu'ils ne se résument pas aux jugements sur des rythmes et des valeurs constituant une émotion esthétique, et pas davantage aux seules relations que les choses objectifient, soit en circulant soit parce qu'on leur « délègue » - au sens de Bruno Latour ${ }^{48}$ - la tâche de faire respecter des obligations sociales.

Outre les travaux de Mahias, Martinelli ou Lemonnier, directement marqués par LeroiGourhan et son école ${ }^{49}$, des recherches de plus en plus nombreuses ont modifié le statut de l'analyse ethnologique des techniques et des objets modestes qui leur sont associés. Prêter la plus grande attention aux actions sur la matière en train de se faire ou à l'utilisation physique des objets révèle désormais des aspects fondamentaux des organisations sociales et des systèmes de pensée qu'il n'est pas possible de mettre en évidence autrement. En voici trois exemples. 
structurales dans les Massim, Damon a donc ajouté l'étude des embarcations utilisées pour la navigation d'île en île. Or, à y regarder de près, et pour peu d'y consacrer les mois et années nécessaires à leur compréhension, ainsi que Mauss le demandait dans ce que Schlanger a appelé un programme "utopique », les matériaux, les assemblages et les équilibres mécaniques pensés, utilisés et réalisés lors de la fabrication d'une pirogue traduisent des relations sociales entre les îles. "Pour l'observateur capable d'en comprendre l'usage ", comme disait Lévi-Strauss à propos d'outils de pierre ${ }^{51}$, les pirogues deviennent des révélateurs de pans entiers de la culture locale. Ce que commentent les producteurs, utilisateurs ou simples observateurs des esquifs, ce ne sont pas seulement les célébrissimes objets du kula - voir « l'enchantement » à distance que créerait la décoration des embarcations, telle qu'envisagés par Gell ${ }^{52}-$, ce sont aussi les types d'arbres utilisés et les liens qui unissent les pièces qu'on en tire, les proportions du mât et la distance de la coque au flotteur, la faculté de ces derniers à amortir les rafales de vent et les vagues. Cette chose matérielle qu'est une " pirogue " apparaît alors comme une synthèse complexe d'un monde social dispersé en mer et associant explicitement l'idée de réseau interîles à une gestion du risque de famine ou de destruction des sources d'approvisionnement en matériaux - un risque de chaos, dit Damon - lié au phénomène El Niño. Autant de domaines de pensée et d'action des populations du Kula ring qui seraient restés inconnus, et dont la compréhension n'ouvre pas que des perspectives nouvelles sur cet ensemble des sociétés. Comme on le voit, c'est aussi le domaine de recherche intuitivement défriché par Francastel ou LeroiGourhan qui se trouve abordé, dans les termes mêmes prônés par ces auteurs.

47 L'étude de Ludovic Coupaye sur la production des grandes ignames cérémonielles des Abelam ${ }^{53}$ constitue une autre illustration de ces travaux qui renouvellent notre compréhension des objets et de leur implication dans le monde de ceux qui les produisent, utilisent, regardent, échangent, etc., en l'occurrence celle des masques dits «à ignames » des Abelam du Sepik (Papouasie Nouvelle-Guinée). La région concernée se trouve, là encore, si ce n'est à l'origine d'un domaine anthropologique entier, du moins à la source d'un foisonnement d'idées nouvelles en anthropologie de l'art, sous la plume de Forge, dont on se souvient qu'il a proposé une approche des peintures et sculpture centrée sur leurs modes d'efficacité rituelle ${ }^{54}$.

Mettant en œuvre la méthode de Leroi-Gourhan, c'est-à-dire en faisant sienne cette foi dans le recueil de chânes opératoires qui caractérise la technologie culturelle développée par deux de ses élèves, Balfet et Cresswell $^{55}$, Coupaye a logiquement remis sur le devant de la scène les ignames qui passionnent les Abelam et étudié dans le plus grand détail la mise en culture et de décoration de ces tubercules, qu'il a considérées comme des «artefacts». Ce faisant, il a montré que leur production impliquait des 
réseaux d'échanges (de boutures, de magies, de monnaies, d'expertises) et une rivalité entre individus et groupes du type de ceux que l'on rencontre ailleurs en NouvelleGuinée, mais à propos de porcs, de têtes coupées ou d'objets précieux. À sa manière, et au prix d'une enquête sur l'agriculture sans précédent, il a lui aussi prouvé combien sont entrelacés la technique la plus matérielle, les rituels, les échanges et toute la vie sociale dans cette partie du monde. Nul doute que Francastel ou Leroi-Gourhan seraient émerveillés d'apprendre que c'est précisément cet entrelacement qui octroie aux grandes ignames leur "pouvoir esthétique ", leur capacité à agir sur leur public lors des cérémonies, et en fait des richesses qu'on peut échanger.

Dans sa recherche consacrée aux sculpteurs chez les Owa de Aorigi (îles Salomon) et aux objets de bois qu'ils produisent, Sandra Revolon montre elle aussi le chemin récemment parcouru dans l'étude du rôle des objets dans les organisations sociales et les systèmes de pensée dès lors que l'on étudie ensemble les arts et les techniques. Cette étude de cas est d'autant plus spectaculaire que les objets auxquels ce chercheur a consacré une ethnographie de longue durée avaient auparavant été analysés dans The Social life of Things ${ }^{56}$, ouvrage qui constitua une étape marquante de cette "redécouverte» des objets dont font grand cas ceux qui ignorent... les travaux de Leroi-Gourhan et de ses successeurs.

Il apparaît ainsi que ce qui est localement reconnu (et nommé) comme la «loi » ordonnant toute vie sociale parle aussi bien des critères de la beauté que des échanges entre humains ou entre humains et esprits. Mais, surtout, alors que l'article de William Davenport ${ }^{57}$ traitait des divers "types de valeurs" illustrés par l'économie de la production et de l'usage de bols cérémoniels, Revolon ajoute que cette petite société des îles Salomon distingue le «beau " du «bien fait » et que les esprits sont réputés " aimer le beau $~^{58}$. En écoutant et en regardant les sculpteurs travailler le bois ou les incrustations de nacre qui décorent les objets, ce chercheur montre que, dans l'acte technique même, le façonnage de l'objet met en jeu l'expert autant que les esprits. Autrement dit, ce n'est pas seulement l'objet fini (et son iconographie) et son rôle dans les échanges ou les stratégies économiques et politiques des organisateurs des cérémonies qui importent : c'est la façon dont, matériellement, on incorpore dans les objets un piège à esprits en faisant, de ses mains, à partir d'une bille de bois, un bol qui fascine les habitants du monde invisible. Il reste bien entendu d'actualité de s'interroger sur le prestige de ceux qui échangent, la valorisation des formes innovantes ou l'effort collectif demandé et publiquement montré pour acquérir les bols ou offrir de la nourriture, comme le faisait Davenport dans l'ouvrage d'Appadurai. Mais une autre catégorie d'acteurs éminents s'invite dans l'échange, les esprits, dont le regard imaginé sur les objets et ceux qui les sculptent bouleverse non seulement notre compréhension de la société owa, mais aussi notre compréhension de la production et du rôle d'objets où s'enchevêtrent art et technique ${ }^{59}$. Au passage, qui songerait, après un tel exemple, à distinguer ce qui relève de l'esthétique, de la religion, des rites de mort ou de la rivalité larvée entre experts?

\section{Bouclée, la boucle?}

51 Résumons. Avec ou sans référence directe à Leroi-Gourhan, mais parce qu'elles s'intéressent aussi à l'action sur la matière, ces recherches nous apprennent que monde du kula se retrouve dans une pirogue, qu'un igname est une chose dont la production 
prend des allures de fait social total, et que le geste d'un sculpteur clame sans mots le lien suprême des esprits et du beau. Certes, il est seulement démontré une fois que les objets évoquent à leur manière (et souvent, en leur matière) des aspects prépondérants de l'ordre social et des représentations locales. Mais ce que l'on aperçoit, enfin, c'est aussi la façon dont ces divers aspects de la vie sociale, de ses pratiques et de son imaginaire se rencontrent dans divers objets. Point fondamental après des décennies de passion pour le "style", cette convergence de pensées qu'ils signalent n'est pas seulement donnée à voir ou à "lire " sur ces productions matérielles : elle est un rapport déterminant avec leur fabrication même ou avec leur mise en œuvre.

En leur temps, Francastel et Leroi-Gourhan furent doublement d'avant-garde, même si l'un et l'autre étaient littéralement pétris des idées de Mauss et, semble-t-il, perméables à ceux de leurs contemporains dont les idées ou recherches pouvaient alimenter leur propre réflexion. L'approche de Bertrand Gille en terme de système, de goulots d'étranglement, de paliers de développement technique, par exemple, se lit clairement en filigrane de bien des pages de Francaste ${ }^{60}$. De Leroi-Gourhan aussi, sans doute, mais le préhistorien ayant eu pour les références la légèreté déjà signalée, nous n'en saurons jamais rien!

D'abord, ils furent pionniers par leur volonté de replacer l'art et la technique dans l'action sociale et d'en envisager l'étude comparée à travers le temps et l'espace sans s'en tenir seulement aux rapports sociaux établis à l'occasion de la production ou de l'usage des objets, à la sociologie de leur usage ou de leur production, comme l'anthropologie économique marxiste d'antan, les material culture studies ou la sociologie de l'innovation excellent d'ailleurs à faire. Ici, il est possible que leur passion pour la matière et ses transformations physiques, qu'il s'agisse de viser des efficacités matérielles ou des esthétiques, les ait empêchés de s'enliser, comme d'autres l'ont fait, dans une application étroite du marxisme aux sociétés non capitalistes, c'est-à-dire en s'attachant aux seuls rapports que les hommes nouent entre eux à propos des objets, sans se demander comment les choses sont fabriquées et physiquement utilisées.

Avec le recul, et bien que la distinction entre art et technique prônée par nos auteurs ait été historiquement fort brève, il était alors également d'avant-garde de chercher d'emblée à penser ensemble l'art et la technique. Cela était aussi une manière de ne pas établir de solution de continuité entre les objets utilitaires, modestes, et ceux qui, localement, sont commentés pour des raisons qui rappellent celles que nous qualifions d'esthétiques. Comme indiqué à l'instant, la prégnance des idées du moment, largement liée à la pratique de l'archéologie, a orienté les recherches vers le « style ", c'est-à-dire les nuances de formes, de décor ou de matière susceptibles de caractériser un groupe humain, souvent pour le seul observateur, parfois pour le groupe en question. L'accent sur les derniers degrés du fait, auxquels Leroi-Gourhan a limité le «style ethnique » des objets " techniques », a incontestablement retardé cette trouvaille d'importance qu'un simple décalage de l'observation et de l'analyse anthropologique a récemment permise : à savoir la part des actions matérielles elles-mêmes dans l'objectivation non verbale de relations sociales et de représentations essentielles pour ceux qui produisent ou utilisent ces objets. Tout laisse penser que certains objets - entendre: leur fabrication ou leur usage physique - ont pour fonction de rassembler et mêler des bribes de pensées et d'actions. Il y a même lieu de se demander s'ils ne sont pas le seul moyen dont se dote une culture pour souligner le besoin ultime de les penser ensemble. À travers l'action la plus matérielle se construit le renforcement réciproque d'aspects 
particulier de plusieurs domaines de la réalité sociale. Lesquels sont non seulement compatibles entre eux, mais concourent ensemble à l'existence et à l'expression d'une même logique sociale, caractéristique d'un groupe donné ${ }^{61}$. Nous sommes alors, enfin, au cœur des intuitions et du programme commun à Francastel et Leroi-Gourhan, mais libérés de l'obsession stylistique.

\section{BIBLIOGRAPHIE}

Appadurai 1986 : Arjun Appadurai, dir., The Social Life of Things, New York/Cambridge, Cambridge University Press, 1986.

Balfet 1975 : Hélène Balfet, « La Technologie », dans Robert Cresswell, dir., Élements d'ethnologie, 2 six approches, Paris, A. Colin, p. 44-47.

Balfet 1991 : Hélène Balfet, Observer l'action technique. Des chaînes opératoires pour quoi faire? Paris, CNRS, 1991.

Balfet 1993 : Hélène Balfet, « Tendance et analyse des documents matériels », Techniques et Culture, 1993, 21, p. 27-36.

Boas 1927 : Franz Boas, Primitive Art, Oslo, Instituttet for sammelignende kulturforshning, 1927. Bonnemère 1996 : Pascale Bonnemère, Le Pandanus rouge. Corps, différence des sexes et parenté chez les Ankave-Anga, Paris, CNRS Éditions/Éditions de la Maison des sciences de l'homme, 1996.

Cresswell 1975 : Robert Cresswell, Éléments d'ethnologie 2, six approches, Paris, Armand Colin, 1975.

Cresswell 2002 : Robert Cresswell, « Geste technique, fait social total. Le technique est-il dans le social ou face à lui », Techniques et Culture, 2002, 40, p. 125-151.

Coupaye 2004 : Ludovic Coupaye, « Growing Artefacts, Displaying Relationships: Outlining the technical system of Long Yam cultivation and display among the Abelam of Nyamikum village (East Sepik Province, Papua New Guinea) », PhD diss., Norwich, University of East Anglia, 2004.

Coupaye 2007a : Ludovic Coupaye, « Des portraits Abelam », Arts \& Cultures, 2007, p. 258-75.

Coupaye 2007b : Ludovic Coupaye, « Beyond Mediation : The Long Yams of Papua New Guinea », dans Celina Jeffrey, Gregory Minissale, dir., Art Histories : Global and Local Mediations, Cambridge, Cambridge Scholar Press, p. 205-220.

Damon (à paraître) : Frederick Damon, On the Ideas of a Boat : From Forest Patches To Cybernetic Structures In The Outrigger Sailing Craft of The Eastern Kula Ring, Papua New Guinea, à paraitre.

Davenport 1986 : William H. Davenport, « Two Kinds of Value in the Eastern Solomon Islands », dans Arjun Appadurai, dir., The Social Life of Things, New York/Cambridge, Cambridge University Press, p. 95-109.

Demoule 2005 : Jean-Paul Demoule, « Archéologie, style et société », dans Bruno Martinelli, dir., L'Interrogaton du style. Anthropologie, technique et esthétique, Aix-en-Provence, Presses de l'université de Provence, p. 49-66. 
Febvre 1947 : Lucien Febvre, « Essor de la découverte », dans André Leroi-Gourhan, dir., Les Explorateurs célèbres, Genève, Mazenod, 1947, p. 34-39.

Forge 1970: Anthony Forge, « Learning to see in New Guinea? », dans Philip Mayer, dir., Socialization: The Approach from Social Anthropology, Londres, Tavistock (ASA Monographs 8), p. 269-291.

Forge 1973 : Anthony Forge, « Style and meaning in Sepik art », dans Anthony Forge, Primitive Art and Society, Londres, Wenner-Gren \& Oxford University Press, p. 169-192.

Francastel 1948 : Pierre Francastel, «Technics and Aesthetics ", The Journal of Aesthetics and Art Criticism, 11 (3), p. 187-197 [traduction de « Technique et Esthétique », Cahiers Internationaux de Sociologie, 5, 1948, p. 97-116].

Francastel 1956 : Pierre Francastel, Art et Technique au XIX et XXe siècles. Paris, Gallimard, 1956.

Francastel 1968 : Pierre Francastel, « Esthétique et ethnologie », dans Jean Poirier, dir., Ethnologie générale, Paris, NRF/Gallimard, 1968, p. 1706-1729.

Francastel 1970 : Pierre Francastel, études de sociologie de l'art, Paris, Denoël-Gonthier Gell 1998 : Alfred Gell, Art and Agency. An Anthropological Theory, Oxford, Clarendon Press, 1998. Gell 2009 : Alfred Gell, L'Art et ses agents, une théorie anthropologique, Bruxelles, Les presses du réel (« Fabula »), 2009.

Gille 1947 : Bertrand Gille, Les Origines de la grande industrie métallurgique en France. Paris, DomatMontchrétien, 1947.

Gille 1964 : Bertrand Gille, Les Ingénieurs de la Renaissance, Paris, Hermann, 1964.

Gille 1978 : Bertrand Gille, Histoire des techniques, Paris, NRF/Gallimard, 1978.

Gille 1980 : Bertrand Gille, Les Mécaniciens grecs. La naissance de la technologie, Paris, Seuil, 1980.

Kaufmann 2005 : Christian Kaufmann, «Canon et Style. Diversité stylistique dans l'art kwoma de Papouasie Nouvelle-Guinée », dans Bruno Martinelli, dir., L'Interrogaton du style. Anthropologie, technique et esthétique, Aix-en-Provence, Presses de l'université de Provence, p. 67-83.

Latour 1993 : Bruno Latour, La Clef de Berlin et autres leçons d'un amateur de sciences, Paris, La Découverte, 1993.

Lemonnier 1993 : Pierre Lemonnier, dir., Technological Choices. Transformation in Material Cultures since the Neolithic, Londres, Routledge, 1993.

Lemonnier 2004 : Pierre Lemonnier, «Les gestes en paroles des esprits cannibales anga (Papouasie Nouvelle-Guinée) », dans Françoise Audouze, Nathan Schlanger, dir., Autour de l'homme. Contexte et actualité d'André Leroi-Gourhan, Antibes, APDCA, p. 163-174.

Lemonnier 2005 : Pierre Lemonnier, « Mythiques chaînes opératoires ", Techniques et culture, 2005, 43-44, p. 25-43.

Lemonnier 2006 : Pierre Lemonnier, Le Sabbat des lucioles. Sorcellerie, chamanisme et imaginaire cannibale en Nouvelle-Guinée, Paris, Stock, 2006.

Leroi-Gourhan 1943 ; André Leroi-Gourhan, Documents sur l'art comparé de l'Eurasie Septentrionale, Paris, Éditions d'art et d'histoire, 1943.

Leroi-Gourhan 1965 : André Leroi-Gourhan, Le Geste et la Parole. La mémoire et les rythmes. Paris, Albin Michel, 1965. 
Leroi-Gourhan [1943] 1971 : André Leroi-Gourhan, Évolution et Techniques. L'homme et la matière. Paris, Albin Michel, [1943] 1971.

Leroi-Gourhan [1945] 1973 : André Leroi-Gourhan, Évolution et Techniques. Milieu et technique. Paris, Albin Michel, [1945] 1973.

Leroi-Gourhan 1982 : André Leroi-Gourhan, Les Racines du monde. Entretiens avec Claude-Henri Roquet. Paris, Belfond, 1982.

Leroi-Gourhan 1983 : André Leroi-Gourhan, Le fil du temps, Paris, Fayard, 1983.

Bernot 1988 : Lucien Bernot, dir., André Leroi-Gourhan ou les voies de l'homme (Actes du colloque du CNRS - mars 1987), Paris, Albin Michel, 1988.

Lévi-Strauss 1966 : Claude Lévi-Strauss, Leçon inaugurale faite le mardi 5 janvier 1960 par M. Claude Lévi-Strauss, professeur, Paris, Collège de France, 1966 (Claude Lévi-Strauss, Anthropologie structurale II, Paris, Plon, p. 11-44).

Lévi-Strauss 1988 : Claude Lévi-Strauss, « ... nous avons lui et moi essayé de faire à peu près la même chose ", dans Lucien Bernot, dir., André Leroi-Gourhan ou les voies de l'homme (Actes du colloque du CNRS - mars 1987), Paris, Albin Michel, 1988, p. 201-206.

Martinelli 2005 : Bruno Martinelli, «Style, technique et esthétique en anthropologie », dans Bruno Martinelli, dir., L'Interrogation du style. Anthropologie, technique et esthétique, Aix-enProvence, Presses de l'université de Provence, p. 19-66.

Mauss [1947] 1967 : Marcel Mauss, Manuel d'ethnographie Paris, Payot (« Petite bibliothèque Payot »), [1947] 1967.

Mahias 1993 : Marie-Claude Mahias, « Pottery Techniques in India. Technical variants ans social choices ", dans Pierre Lemonnier, dir., Technological Choices. Transformation in Material Cultures since the Neolithic, Londres, Routledge, 1993, p. 157-180.

Mahias 2002 : Marie-Claude Mahias, Le Barattage du monde. Essais d'anthropologie des techniques en Inde, Paris, MSH, 2002.

Meyerson 1953 : Ignace Meyerson, « Intervention dans la discussion des exposés de Bertrand Gille, "Lents progrès de la technique" et de Pierre Francastel, "Techniques et arts", Revue de synthèse, 1953, 32, n. s., p. 109-111.

Rappaport 1979 : Roy Rappaport, Ecology, Meaning, Religion, Richmond (Ca), North Atlantic Book, 1979.

Revolon 2006 : Sandra Revolon, « Manira (Aorigi, Est des îles Salomon) », Cahiers d'anthropologie sociale, 2006,1, p. 97-111.

Revolon 2007a : Sandra Revolon, « "Les esprits aiment ce qui est beau”. Formes, sens et efficacité rituelle des sculptures owa (Est des îles Salomon) ». Annales de la Fondation Fyssen, 2007,21, p. 63-75.

Revolon $2007 b$ : Sandra Revolon, « Sacré curios. Du statut changeant des objets dans une société mélanésienne (Aorigi, est des îles Salomon) », Gradhiva, 2007, nº 6, p. 59-71.

Schaeffer 2004 : Jean-Marie Schaeffer, « Objets esthétiques », L’Homme, 2004, 170, p. 25-45.

Schlanger 1991 : Nathan Schlanger, « Le Fait technique total : la raison pratique et les raisons de la pratique dans l'œuvre de Marcel Mauss », Terrain, 1991, 16, p. 114-130. 


\section{NOTES}

1. Francastel, 1970, p. 11.

2. Leroi-Gourhan 1943b, p. 16.

3. Francastel 1988 (1956), p. 107.

4. Francastel 1988 (1956), p. 110.

5. Leroi-Gourhan 1983, p. 7.

6. Leroi-Gourhan 1971 (1943), p. 13.

7. Francastel 1988 (1956).

8. Leroi-Gourhan 1971 (1943).

9. Leroi-Gourhan 1983, p. 16.

10. Leroi-Gourhan 1983, p. 31-32.

11. Francastel 1970, p. 27.

12. Francastel 1953, p. 188.

13. Francastel 1956, p. 12.

14. Francastel 1953, p. 189.

15. Febvre 1947.

16. Francastel 1956, p. 121.

17. Meyerson 1953.

18. Francastel 1968, p. 1713.

19. «L'esthétique contribue à l'efficacité, aussi bien que les rites (le nombre des objets purement laïques serait assez restreint) » (Mauss 1967, p. 86).

20. «Le but de l'art n'est pas de constituer un double maniable de l'univers; il est à la fois de l'explorer et de l'informer d'une manière nouvelle » (Francastel 1956, p. 12).

21. Lucien Bernot 1988, p. 205.

22. Mauss 1967, p. 85. Ou de manière plus concise encore : « Où trouver de l'esthétique ? D'abord, dans l'ensemble des techniques... » (Mauss 1967, p. 86).

23. Francastel 1956, p. 7 .

24. Boas 1927.

25. «Dès qu'apparaît la plastique, on voit surgir des notions d'équilibre, donc des notions de rythme ; et dès qu'apparaît la rythmique, l'art apparaît » (Mauss 1967, p. 85) ; «Boas, dans son Primitive Art [1927], rattache tout l'art au rythme : car là où il y a rythme, généralement il y a esthétique ; là où il a des tons, des variations de touches et d'intensité, il y a généralement esthétique " (Mauss 1967, p. 87). Sur la construction de la notion de style empruntée par les ethnologues à l'histoire de l'art, voir Kaufmann 2005 et, sur ses emplois divers par Boas, Kroeber, Mauss ou Leroi-Gourhan, voir Martinelli 2005.

26. Leroi-Gourhan 1982, p. 172.

27. Schlanger 1991.

28. Leroi-Gourhan 1982, p. 38.

29. Leroi-Gourhan 1973.

30. Francastel 1956, p. 44.

31. Lemonnier 1993.

32. Schlanger 1991.

33. Francastel [1948] 1953, p. 190.

34. Francastel 1968, p. 1721-1724.

35. Leroi-Gourhan 1971 ; Leroi-Gourhan 1973.

36. Lévi-Strauss 1988, p. 205.

37. Mauss 1967, p. 22, 27, 32 et 35. 
38. Le cadre d'analyse de Leroi-Gourhan «s'appuie sur les caractères physiques de la matière elle-même [...]. Ce sont les matériaux et les moyens d'action sur la matière qui conditionnent tout le reste » (Leroi-Gourhan 1982, p. 34).

39. Appadurai 1986.

40. Francastel 1968, p. 1723.

41. "La collection des types d'une population déterminée donne la notion de son style et de sa singularité esthétique [...]. L'ensemble des éléments esthétiques qui caractérisent une société constitue le style de cette société » (Mauss 1967, p. 105)

42. Leroi-Gourhan 1965, p. 93.

43. "Mais alors que les figures verbales, dans les mots et dans la syntaxe, sont comme l'équivalent des outils et des gestes manuels, destinés à assurer une prise efficace sur le mode de la matière et des relations, la figuration se fonde sur un autre champ biologique qui est celui de la perception des rythmes et des valeurs, commun à tous les êtres vivants. Outils, langage et création rythmique sont donc bien trois aspects contigus du même processus » (Leroi-Gourhan 1965, p. 210).

44. Schaeffer 2004.

45. Demoule 2005.

46. Lemonnier 1993.

47. Mahias 1993.

48. Latour 1993.

49. Mahias 2002 ; Martinelli 2005 ; Lemonnier 2005 ; Lemonnier 2006, p. 203-273.

50. Damon (à paraître).

51. Lévi-Strauss 1966, p. 15.

52. Gell 1998.

53. Coupaye 2004 ; Coupaye 2007a ; Coupaye $2007 \mathrm{~b}$.

54. Forge 1970 ; Forge 1973.

55. Cresswell 1975 ; Balfet 1991 ; Balfet 1993 ; Cresswell 2002.

56. Appadurai 1986.

57. Davenport 1986.

58. Revolon 2006 ; Revolon 2007a.

59. Revolon $2007 \mathrm{~b}$.

60. Francastel 1968, p. 1722.

61. Lemonnier 2004, p. 173.

\section{RÉSUMÉS}

Comparer deux esprits dissemblables, qui se sont intéressés à des champs différents, peut sembler un exercice d'école. Cependant, l'œuvre de ces deux savants a marqué de façon si forte la réflexion contemporaine sur les origines de l'art qu'elle nous paraît ouvrir un chapitre singulier de l'histoire des sciences humaines en France. Car, malgré la diversité de leurs parcours et de leurs méthodes, Francastel et Leroi-Gourhan ont été les théoriciens et les praticiens d'une approche de l'art qui fait de la matière et des techniques le socle de l'interprétation des œuvres. Francastel est né en 1900, Leroi-Gourhan en 1911, l'un et l'autre ont commencé très jeunes leur carrière dans des fonctions atypiques, attaché comme Francastel à la direction des travaux de 
Versailles, boursier comme Leroi-Gourhan au musée Guimet et au CNRS. Les premiers travaux de Francastel portent sur la sculpture à Versailles, ceux de Leroi-Gourhan sur le bestiaire du bronze chinois. Travaux de débutants qui les montrent à pied d'œuvre sur les collections des musées, attentifs à la connaissance directe des objets mais intéressés dès le départ à leur condition de création et de réception. Francastel est fasciné par la peinture et les arts de l'Occident, LeroiGourhan est attiré par le monde oriental dont il domine très tôt les langues et les arts. Tous deux entrent à l'université par des chemins de traverse, tous deux effectuent de longs séjours à l'étranger, en Pologne pour Francastel, au Japon pour Leroi-Gourhan.

Passionnés par la matérialité des choses, attentifs aux techniques et aux objets utiles, LeroiGourhan et Francastel ont refusé l'opposition entre technique et esthétique, entre objet modeste et œuvre d'art. Ils ont souligné que l'art est une technique "opératoire et figurative " (Francastel) que l'on ne peut comprendre sans saisir ses liens matériels et mentaux avec la culture matérielle (ou le système technique) du moment.

Comparatistes forcenés, attachés aux coévolutions et aux systèmes, ils ont, chacun à leur manière, ancré l'étude des rapports de l'art et de la technique dans une sociologie et une histoire allant bien au-delà de ces domaines particuliers de la vie sociale. Ces pionniers rappellent à ceux qui en douteraient que l'on ne peut comprendre une œuvre d'art sans se pencher tout autant sur les objets modestes. Y compris pour aborder ces questions nouvelles que soulèvent, par exemple, le rôle sui generis des objets dans l'action rituelle ou l'inscription d'une préoccupation « esthétique » dans la structure même des objets, et non pas dans leur décor.

\section{AUTEURS}

\section{ALAIN SCHNAPP}

Centre national de la recherche et Université Paris-I

Alain Schnapp est spécialiste d'archéologie grecque. Il a dirigé des chantiers de fouilles en Italie et en Grèce et s'est intéressé à l'histoire et l'interprétation des images en Grèce ancienne. Ses travaux récents portent sur l'histoire de l'archéologie et la réception du passé en Occident et en Orient. Il a enseigné dans de nombreuses universités étrangères et, après avoir été le premier directeur de l'Institut national d'histoire de l'art, il a repris ses fonctions de professeur à l'université Paris I depuis 2005.

Publications récentes :

- Préhistoire et antiquité (éd.), Paris, Flammarion (« Histoire de l'art », vol. I), 1997.

- La conquête du passé, Paris, Le Livre de poche, 1998.

- L'histoire ancienne à travers cent chefs-d'œuvre de la peinture (avec François Lebrette), Paris, Presses de la Renaissance, 2004.

- Guide des méthodes de l'archéologie (avec P. Demoule, F. Giligny et A. Lehoerff), Paris, La

Découverte, 2005.

\section{PIERRE LEMONNIER}

Directeur de recherche au CNRS (Centre de recherche et de documentation sur l'Océanie, CNRSEHESS-université de Provence, Marseille), Pierre Lemonnier a étudié la production de sel de la côte atlantique française (Les salines de l'Ouest, 1980 ; Paludiers de Guérande, 1984), puis celle des Anga de Papouasie-Nouvelle-Guinée. Ses travaux actuels portent sur les rituels des Ankave-Anga et sur la place particulière qu'y tiennent les objets. Outre des ouvrages théoriques sur l'anthropologie des techniques (Elements for an Anthropology of Technology, 1992 ; Technological Choices, 1993 ; L'intelligence des techniques, 1993 [avec Bruno Latour]), il a publié Guerres et festins. Paix, échanges et compétition dans les Hautes Terres de Nouvelle-Guinée (1990) et Le sabbat des lucioles 
(2006), à propos de la sorcellerie, du cannibalisme et du chamanisme chez les Ankave-Anga (où il enquête depuis 1982). 